\title{
Prenatal Omega-3 Supplementation and Eczema Risk among Offspring at Age 36 Months
}

\author{
Berman D ${ }^{1}$, Clinton $C^{2}$, Limb R ${ }^{3}$, Somers EC ${ }^{4,5}$, Romero ${ }^{6}$, Mozurkewich $\mathrm{E}^{7}$ \\ ${ }^{1}$ Department of Obstetrics and Gynecology, Division of Maternal Fetal Medicine, University of Michigan, Ann Arbor, MI, United States \\ ${ }^{2}$ Department of Obstetrics and Gynecology, Cornell University, New York NY, United States \\ ${ }^{3}$ University of Michigan, Ann Arbor, MI, United States \\ ${ }^{4}$ Department of Internal Medicine, University of Michigan, Ann Arbor, MI, United States \\ ${ }^{5}$ Department of Environmental Health Sciences, University of Michigan, Ann Arbor, MI, United States \\ ${ }^{6}$ Department of Obstetrics and Gynecology, Division of Maternal Fetal Medicine, Michigan State University, College of Human Medicine, Grand \\ Rapids, MI, United States
}

${ }^{7}$ Department of Obstetrics and Gynecology, Division of Maternal Fetal Medicine, University of New Mexico, Albuquerque, NM, United States

Corresponding author: Deborah Berman, Department of Obstetrics and Gynecology, Division of Maternal Fetal Medicine, University of Michigan, Ann Arbor, MI, United States, Tel: 734-763-4264; Fax: 734-936-3151; E-mail: debster@umich.edu

Received date: Feb 15, 2016; Accepted date: Apr 02, 2016; Published date: Apr 10, 2016

Citation: Zafar A. Prenatal Omega-3 Supplementation and Eczema Risk among Offspring at Age 36 Months. Insights Allergy Asthma Bronchitis. 2016; 2:1.

\section{Abstract}

\section{Background:}

Long-term follow-up was completed in 84 mother-infant pairs of 118 women who completed a randomized controlled trial of prenatal supplementation with EPA- or DHA-rich fish oil or soy oil placebo. The goal of this study was to determine whether prenatal omega-3 fatty acid supplementation protects offspring against development of early childhood allergies.

\section{Methods and Findings:}

Assessment of childhood allergic/atopic disease among offspring at age 36 months was performed by maternal interview using the National Health Interview Survey (NHIS) questions for childhood digestive allergies, wheezing, eczema or skin allergy, and respiratory allergy. Multiple logistic regressions examined the association between prenatal supplementation and childhood outcomes, adjusted for covariates.

Eczema was reported in 26/84 (31\%) of offspring at age 36 months, and was significantly more prevalent in the omega-3 supplementation groups vs. placebo: EPA 13/31 (41.9\%); DHA $10 / 26$ (38.5\%); placebo $3 / 27$ (11.1\%), $p=0.019$. Compared to placebo, EPA and DHA were associated with $\geq 5$ times risk of offspring eczema [odds ratios (ORs): EPA $5.8(95 \% \mathrm{Cl}$ 1.4-23.3); DHA 5.0 (95\% Cl 1.2-21.0)]. After adjusting for other potential risk factors (race, birth weight, vaginal/ Cesarean delivery, and maternal eczema) the magnitudes of association for omega-3 supplementation increased: EPA OR 8.1 (95\% Cl 1.4-45.6); DHA OR 9.6 (95\% Cl 1.6-58.5). Maternal eczema was also significantly associated with offspring eczema in the adjusted model: OR 10.8 (95\% Cl 2.1-54.3)

\section{Conclusion:}

Contrary to our hypothesis, acids supplementation compared to soy oil was associated with a substantial increase in risk of childhood eczema. This association was not observed on childhood respiratory or digestive outcomes. It is unclear if these findings were driven by unfavorable effects of omega-3s, or whether there may have been unanticipated protective effects of the soybased placebo with regards to eczema.

Keywords: Hypothesis; Allergic diseases; Fatty acids

\section{Introduction}

Allergic conditions are among the most common medical conditions affecting children in the United States. Allergies are the fifth leading cause of chronic disease in the United States [1]. Skin allergies (such as eczema), food or digestive allergies, and respiratory allergies (such as hay fever) are the most common allergies among children. The prevalence of food and skin allergies increased in children aged 0-17 years from 1997-2011 [2].

As allergic diseases are often diagnosed in early infancy, this suggests that causative events take place in utero or during the neonatal period $[3,4]$. It has been hypothesized that maternal diet may impact neonatal immune development and subsequently alter allergic responses of neonates. Specifically, the change in Western diet from a relative balance between anti-inflammatory omega-3 fatty acids and pro-inflammatory omega- 6 fatty acids, to a diet in which omega- 6 fatty acids are overwhelmingly predominant is a possible explanation for the observed increase in the incidence of childhood atopic disease [5]. Intake of omega-3 fatty acids has been shown to suppress cell mediated immune responses in animal and human studies [6]. Specifically, we have previously shown that prenatal 
eicosapentaenoic acid (EPA) and docosahexaenoic acid (DHA)rich fish oil supplementation altered Th2/Th1 chemokine ratios at birth, reducing the predominantly Th2 response that has been reported to promote allergic disease [7].

Observational studies have found higher cord blood DHA status related to lower probability of later development of atopic eczema [8]. A review of four randomized controlled trials with 698 participants found that omega-3 fatty acid supplementation during pregnancy decreases childhood asthma and response to the egg skin prick test [9]. A more recent large randomized study of prenatal omega- 3 fatty acid supplementation found that prenatal DHA supplementation compared with vegetable oil supplementation decreased atopic eczema and sensitization to egg [4]. Similarly, a followup study of 869 children found that DHA supplementation significantly reduced respiratory symptoms among children born to atopic mothers; although, paradoxically, respiratory symptoms were increased among children born to DHAsupplemented non-atopic mothers [10] Our hypothesis that omega-3 fatty acid supplementation may function in neonatal immune modulation is supported by these studies.

We performed this follow-up study to determine whether prenatal omega-3 fatty acid supplementation protects offspring against development of early childhood allergies.

\section{Methods}

This research study was a secondary analysis of The Mothers, Omega-3 \& Mental Health Study (MOMS). The MOMS was a prospective, double blind, placebo-controlled, randomized trial designed to assess whether omega-3 fatty acid supplementation during pregnancy may prevent antenatal and postpartum depressive symptoms among 126 pregnant women at risk for depression. Inclusion criteria included past history of depression, an EPDS (Edinburgh Postnatal Depression Scale) score of 9-19 (at risk for depression or mildly depressed), singleton gestation, a maternal age of 18 years or older, and a gestational age of 12-20 weeks. Randomization was carried out using a random number table maintained in the University of Michigan Investigational Drug Service. Details of the overall study design and study population have been published previously [11]. Institutional Review Board (IRB) approval was obtained prior to the start of the study; written patient consent was obtained for participation in this project.

\section{Study Population}

We performed long-term follow-up of mother-infant pairs who completed a randomized controlled trial of prenatal supplementation with EPA-rich fish oil (1060 mg EPA plus 274 mg DHA), DHA-rich fish oil (900 mg DHA plus $180 \mathrm{mg}$ EPA) or soy oil placebo [11]. Follow-up phone interviews were conducted in 85 of the 118 mothers who completed the trial. Eighty-four singletons were included in this analysis, with one twin set excluded due to between-twin correlation and to avoid over-weighting of the maternal data from this pregnancy. Interviews assessed childhood allergic and atopic disease among the offspring at age 36 months, using questions validated from the 2011 NHIS [2], the following outcomes were assessed by maternal report: childhood food or digestive allergies, asthma or persistent wheezing requiring treatment, eczema/skin allergy/atopic dermatitis/chronic rashes, respiratory or nasal allergy, or recurrent croup. Additional information was gathered regarding the presence of maternal, paternal, and sibling allergies. Eczema was defined as an acute or chronic inflammation of the skin, characterized by redness, itching, and the outbreak of lesions that become encrusted and scaly and may discharge serous matter. Food or digestive allergy was defined as an abnormal response to a food resulting in itching or swelling in the mouth, vomiting, diarrhea, or abdominal cramps and pain, hives or eczema, tightening of the throat or trouble breathing, and/or hypotension. Asthma or persistent wheezing was defined as a chronic long-lasting inflammatory disease of the airways, resulting in airway spasm and/or periodic swelling/narrowing, wheezing, chest tightness, shortness of breath, and/or coughing.

Table 1 Characteristics by treatment arm; outcomes at age 36 months.

\begin{tabular}{|c|c|c|c|c|}
\hline & \multicolumn{4}{|c|}{ Treatment Arm } \\
\hline & $\begin{array}{l}\text { Placebo } \\
(n=27)\end{array}$ & $\begin{array}{l}\text { EPA } \\
(n=31)\end{array}$ & $\begin{array}{l}\text { DHA } \\
(n=26)\end{array}$ & $\begin{array}{l}\text { p- } \\
\text { valu } \\
e^{*}\end{array}$ \\
\hline Sex of Child & & & & NS \\
\hline Female & $8(30.8)$ & $16(53.3)$ & $14(53.9)$ & \\
\hline Male & $18(69.2)$ & $14(46.7)$ & $12(46.2)$ & \\
\hline Race & & & & NS \\
\hline White & $26(96.3)$ & $27(87.1)$ & $23(88.5)$ & \\
\hline Other & $1(3.7)$ & $4(12.9)$ & $3(11.5)$ & \\
\hline $\begin{array}{l}\text { Birthweight }(\mathrm{g}) \text {, mean } \\
\text { (SD) }\end{array}$ & $\begin{array}{l}3308.8 \\
(561.5)\end{array}$ & $\begin{array}{l}3387.1 \\
(560.9)\end{array}$ & $\begin{array}{l}3806.6 \\
(444.2)\end{array}$ & NS \\
\hline Mode of delivery & & & & NS \\
\hline Vaginal & $20(74.1)$ & $23(74.2)$ & $17(68.0)$ & \\
\hline Cesarean & 7 (25.9) & $8(25.8)$ & $8(32.0)$ & \\
\hline Maternal eczema & $4(14.8)$ & $6(19.4)$ & $4(15.4)$ & NS \\
\hline $\begin{array}{l}\text { Outcomes (age } 36 \\
\text { months) }\end{array}$ & & & & \\
\hline Eczema/Skin & $3 / 27(11.1)$ & $\begin{array}{l}13 / 31 \\
(41.9)\end{array}$ & $\begin{array}{l}10 / 26 \\
(38.5)\end{array}$ & $\begin{array}{l}0.01 \\
9\end{array}$ \\
\hline Food allergies & $3 / 27(11.1)$ & $\begin{array}{l}4 / 30 \\
(13.3)\end{array}$ & $5 / 26(19.2)$ & NS \\
\hline Asthma/Wheezing & $7 / 27(25.9)$ & $\begin{array}{l}9 / 31 \\
(29.0)\end{array}$ & $4 / 26(15.4)$ & NS \\
\hline Respiratory & $4 / 27(14.8)$ & $\begin{array}{l}4 / 31 \\
(12.9)\end{array}$ & $3 / 26(11.5)$ & NS \\
\hline
\end{tabular}

\section{Statistics}

Characteristics of the study population were summarized according to treatment arm, and presented as frequency 
(proportion) or mean (SD). One-way ANOVA (continuous variables) or Fisher's exact test (categorical variables) were used to compare groups. Separate logistic regression models were used to assess the relationship between treatment arm and each of the four types of allergy/atopy outcomes. Multiple logistic regressions were performed to further examine the association between prenatal supplementation and eczema, adjusting for covariates determined a priori as potentially relevant. These a priori variables were included even if distributed similarly between groups, in order to account for the potential of residual confounding. The final adjusted model included: sex of child, race of child, birthweight (g), mode of delivery (vaginal or Cesarean delivery), and maternal eczema. Power calculations were not performed, given the nature of this study as a secondary analysis and that concerns exist related to post hoc power calculations [12].

\section{Results}

The overall characteristics of the study population were similar between treatment groups (Table 1). There were a smaller proportion of females $(30.8 \%)$ vs. males in the placebo group, though it was not statistically significant. The primary outcomes at 36 months were similar and did not significantly differ between groups for three of the four categories: food allergies, asthma/wheezing, and respiratory allergies. Eczema was reported in 26/84 (31\%) of all offspring at age 36 months and there was a higher rate of eczema in the EPA and DHA arms compared to placebo ( $p=0.019)$ : EPA 41.9\% (13/31), DHA $38.5 \%(10 / 26)$, and placebo $11.1 \%$ (3/27). Compared to placebo, EPA and DHA were associated with a greater than five-fold increased risk of offspring eczema in both the crude (unadjusted) and adjusted models; Crude odds ratios (ORs): EPA 5.8 (95\% Cl 1.4-23.3); DHA 5.0 (95\% Cl 1.2-21.0) (Table 2).

After adjusting for other potential risk factors (sex, race, birthweight, mode of delivery, maternal eczema), risk of eczema was >eight-fold greater for EPA [odds ratio (ORadj)-8.1, 95\% Cl 1.4-46.3] and DHA [ORadj=9.5 (1.5-59.6)] (Table 2). In the adjusted model, maternal eczema was associated with a 10.8 -fold increase in risk of eczema in the offspring $195 \% \mathrm{Cl}$ 2.1-54.3). The remaining variables were not detected to be associated with risk of eczema in either the crude or adjusted models.

Table 2 Multivariable logistic regression with select covariates for outcomes of eczema at age 36 months.

\begin{tabular}{|c|c|c|c|c|}
\hline & Crude Models OR $(95 \% \mathrm{Cl})$ & p-value & Adjusted Model* OR $(95 \% \mathrm{Cl})$ & p-value \\
\hline \multicolumn{5}{|l|}{ Treatment Arm } \\
\hline Placebo & referent & --- & referent & --- \\
\hline EPA & $5.8(1.4-23.3)$ & 0.014 & $8.1(1.4-46.3)$ & 0.019 \\
\hline $\mathrm{DHA}$ & $5.0(1.2-21.0)$ & 0.028 & $9.5(1.5-59.6)$ & 0.016 \\
\hline Sex of Child & $0.9(0.4-2.3)$ & NS & $1.0(0.3-3.0)$ & NS \\
\hline \multicolumn{5}{|l|}{ Race of Child } \\
\hline White & referent & --- & referent & --- \\
\hline Other & $0.7(0.1-3.8)$ & NS & $1.2(0.2-8.4)$ & NS \\
\hline Birth weight (g) & 1 & NS & $1.0(1.0-1.0)$ & NS \\
\hline \multicolumn{5}{|l|}{ Mode of Delivery } \\
\hline Vaginal & referent & --- & referent & -- \\
\hline Cesarean & $0.7(0.2-2.1)$ & NS & $0.6(0.2-2.4)$ & NS \\
\hline Maternal eczema & $5.6(1.7-19.0)$ & 0.006 & $10.8(2.1-54.3)$ & 0.004 \\
\hline
\end{tabular}

\section{Discussion}

We found that prenatal supplementation with omega-3 fatty acids had no effect on most measures of allergic disease at 36 months, which was contrary to our hypothesis. The background rate of self-reported maternal eczema in our study was consistent with the background prevalence in adults: in population-based studies, $17.1 \%$ report at least one of four eczematous symptoms [13]. Contrary to expectation; we found an increased incidence of atopic eczema in children born to supplemented mothers. Our results differ from prior studies, including the conclusions of the Cochrane systematic review that evaluated the effect of omega-3 fatty acid supplementation on allergic outcomes [14]. There may be several reasons for this observed difference.

Previous studies have varied in study design, including timing, length, and dose of supplementation, as well as length of subsequent follow-up for allergy assessment. One of the prior studies demonstrated a decrease in the prevalence of wheeze when supplementation was initiated postnatal and only in high-risk children (defined by affected parents or siblings) [15]. Another study provided antenatal supplementation; however, all supplemented women had allergic disease, compared to our population, which included women both with and without history of allergic disease [16]. 
A meta-analysis of omega- 3 and omega- 6 oils for the primary prevention of allergic disease did not establish a clear trend related to early life fatty acid supplementation and risk of allergic disease [17]. However, although results were not statistically significant, the directions of association were in line with our findings: pooled results from the meta-analysis found a non-significant increase in risk of eczema with omega-3 supplementation but a decrease in risk of other types of allergy, as well as a non-significant protective effect of omega- 6 supplementation against eczema. As our placebo soy oil supplement was omega- 6 fatty acid predominant, it may have exerted a protective effect. More recently, results from a follow-up study of a large randomized controlled trial suggested that the effect of prenatal omega-3 fatty acid supplementation may differ among children born to atopic versus non-atopic mothers.10 It is important to note that the heritability of asthma is on the order of $60 \%-80 \%$ [18], thus the genetic component of atop may be significant.

Strength of our study is that the treatment allocation groups remained blinded at the time the follow-up questionnaire was administered. Strength is that we were able to contact the majority of our participants at 36 months. Additionally, participants were not selected based on predisposition to allergic disease, and we were also able to obtain information on maternal, paternal, and sibling allergies.

However, some limitations of this study exist: it was a pilot investigation with a relatively limited sample size; it was not a planned secondary analysis, so the original study was not designed to be powered for this sub-analysis; and formal allergy testing was not conducted in our study, though the parental interview utilized validated NHIS questions. Thus, our results should be viewed as preliminary and requiring confirmation in larger follow-up studies.

Our previous secondary analysis of the MOMS Study [19] found that prenatal supplementation with EPA- and DHA-rich fish oil significantly reduced cord blood chemokine ratios thought to predict childhood development of allergic disease. Thus, although our study showed no benefit for allergic symptoms, it is clear that this same regimen of supplementation altered the neonatal inflammatory response. It is unclear why our findings occurred, but they may further support the hypothesis that genetics and predisposition play an important role in the response to supplementation.

Another factor that may explain our differing findings is the location of the studies, and therefore, the subjects' background dietary fatty acid composition. Both of the aforementioned studies took place in Australia; other trials have included participants from Sweden, Denmark, Hungary, Norway, Germany, and Spain. Variation in dietary habits between countries may result in considerable differences in baseline fatty acid states and subsequent responses to supplementation. For example, in Sweden, the omega-3 to omega- 6 polyunsaturated fatty acid ratio is approximately $5: 1$ [20], in contrast to many other Western countries in which it is estimated to be 20-30:1 [21]. Thus, women's diets in any one study may not necessarily represent that of other Western populations.
The differing doses of supplementation in the available post-supplementation follow-up studies may also represent a potential explanation for the wide range of findings. Omega-3 fatty acid supplementation may have an inverted U-shaped dose-response curve, with moderate doses conferring more benefit than high doses in some models [22]. It is also possible that linoleic acid, the principal component of the soy placebo, had an unanticipated protective effect on development of atopic eczema-an active placebo effect [23].

It will be important for future studies to consider the dose and timing of supplementation, the background diet fatty acid composition, subjects' genetic predisposition to allergic disease, and the source of omega- 3 fatty acids. These factors appear to all be working together in a manner that has yet to be fully elucidated. Immunomodulation, especially in pregnancy, is complex and has many variables. Future studies are needed to determine the most effective regimen of omega-3 fatty acid supplementation to provide the maximum health benefit. It is clear that omega-3 fatty acid supplementation can affect atopic disease; however, this relationship has proven to be complex and remains to be completely understood.

\section{References}

1. Friedman AH, Morris TL (2006) Allergies and anxiety in children and adolescents: A review of the literature. J Clin Psychol Med Settings 13: 318-331.

2. National Health Interview Survey (2011) U.S. Department of Health and Human Services: Washington, D.C

3. Vassallo MF, Banerji A, Rudders SA, Clark S, Mullins RJ, et al. (2010) Season of birth and food allergy in children. Ann Allergy Asthma Immunol 104: 307-313.

4. Prescott SL, Dunstan JA (2007) Prenatal fatty acid status and immune development: the pathways and the evidence. Lipids 42: 801-810.

5. Blümer N, Renz H (2007) Consumption of omega3-fatty acids during perinatal life: role in immuno-modulation and allergy prevention. J Perinat Med 35 Suppl 1: S12-S18.

6. Simopoulos AP (2002) Omega-3 fatty acids in inflammation and autoimmune diseases. J Am Coll Nutr 21: 495-505.

7. Montes R, Chisaguano AM, Castellote Al, Morales E, Sunyer J, et al. (2013) Fatty-acid composition of maternal and umbilical cord plasma and early childhood atopic eczema in a Spanish cohort. Eur J Clin Nutr 67: 658-663.

8. Palmer DJ, Sullivan T, Gold MS, Prescott SL, Heddle R, et al. (2013) Randomized controlled trial of fish oil supplementation in pregnancy on childhood allergies. Allergy 68: 1370-1376.

9. Klemens CM, Berman DR, Mozurkewich EL (2011) The effect of perinatal omega-3 fatty acid supplementation on inflammatory markers and allergic diseases: a systematic review. BJOG 118: 916-925.

10. Escamilla-Nuñez MC, Barraza-Villarreal A, Hernández-Cadena $L$, Navarro-Olivos E, Sly PD, et al. (2014) Omega-3 fatty acid supplementation during pregnancy and respiratory symptoms in children. Chest 146: 373-382. 
11. Mozurkewich (2013) The Mothers, Omega-3, and Mental Health Study: a double-blind, randomized controlled trial. Am J Obstet Gynecol 208: 1-9.

12. Hoenig J, Heisey D (2001) The abuse of power: The pervasive fallacy of power calculations for data analysis. The American Statistician 55: 1-6.

13. Hanifin JM, Reed ML (2007) A Population-Based Survey of Eczema Prevalence in the United States, Eczema Prevalence and Impact Working Group Disclosures Dermatitis 18: 82-91.

14. Gunaratne AW, Makrides M, Collins CT (1993) Maternal prenatal and/or postnatal n-3 long chain polyunsaturated fatty acids (LCPUFA) supplementation for preventing allergies in early childhood. Cochrane Database of Systematic Reviews Issue 7 London: The Cochrane Collaboration.

15. Mihrshahi S, Peat JK, Marks GB (2003) Eighteen-month outcomes of house dust mite avoidance and dietary fatty acid modification in the Childhood Asthma Prevention Study (CAPS). J Allergy Clin Immunol 111: 162-168.

16. Dunstan J, Mori TA, Barden A (2003) Fish oil supplementation in pregnancy modifies neonatal allergen-specific immune responses and clinical outcomes in infants at high risk of atopy: a randomized controlled trial. J Allergy Clin Immunol 112: 1178-1184.
17. Anandan C, Nurmatov U, Sheikh A (2009) Omega 3 and 6 oils for primary prevention of allergic disease: systematic review and meta-analysis. Allergy 64: 840-848.

18. Holgate ST, Holloway JW (2002) The Hereditary Basis of Allergic Disease. Basel: Birkauser Verlag p: 198.

19. Romero VC (2013) Developmental programming for allergy: a secondary analysis of the Mothers, Omega-3 \& Mental Health Study. Am J Obstet Gynecol 208: 316.e1-316.e6.

20. Furuhjelm C, Warstedt K, Larsson J, Fredriksson M, Böttcher MF, et al. (2009) Fish oil supplementation in pregnancy and lactation may decrease the risk of infant allergy. Acta Paediatr 98: 1461-1467.

21. Simopoulos AP, Leaf A, Salem N (1999) Workshop on the essentiality of and recommended dietary intakes for omega- 6 and omega-3 Fatty acids. Asia Pac J Clin Nutr 8: 300-301.

22. Berman DR, Mozurkewich E, Liu Y, Barks J (2009) Docosahexaenoic acid pretreatment confers neuroprotection in a rat model of perinatal cerebral hypoxia-ischemia. Am J Obstet Gynecol p: 305.

23. Odeleye OE, Watson RR (1991) Health implications of the $n-3$ fatty acids. Am J Clin Nutr 53: 177-178. 